\title{
Symmetries in optical wavefields
}

\author{
J. Jahns
}

J. Jahns, "Symmetries in optical wavefields," Proc. SPIE 11207, Fourth International Conference on Applications of Optics and Photonics, 1120702 (3 October 2019); doi: 10.1117/12.2523115

SDIE Event: IV International Conference on Applications of Optics and Photonics (AOP 2019), 2019, Lisbon, Portugal 


\title{
Symmetries in optical wavefields
}

\author{
J. Jahns * \\ FernUniversität in Hagen, Chair of Micro- and Nanophotonics \\ Universitätsstr. 27, 58097 Hagen, Germany
}

\begin{abstract}
Symmetries in the propagation of a wavefield occur due to constraints, imposed either by the structure of an optical element/system or by the propagation medium. The spatial properties of a wavefield may be influenced by mirror symmetry, lateral inversion, translational and rotational symmetry. Here, we discuss various examples of light propagation under the constraints of specific symmetries. These include general aspects of light propagation, the design of micro-optical systems, rotational symmetries that occur in discretized diffractive optical elements as well as spatial and spatio-temporal properties of self-imaging wavefields.
\end{abstract}

Keywords: optical propagation, symmetry, systems design, diffraction.

1.

\section{INTRODUCTION}

Symmetries are ubiquitous in daily life and play an important role in all branches of the natural sciences ${ }^{1}$. Principles of symmetry are fundamental to theoretical physics and mathematics. Symmetries are at the origin of the conservation laws of physics, for example. However, the violation of symmetry is also built into physics at various levels, most well-known is the violation of parity in beta decay. Here, we focus on symmetries occuring in and affecting optical wavefields. The spatial properties of a wavefield may be influenced by mirror symmetry (longitudinal inversion), lateral inversion, translational and rotational symmetry. The temporal properties may also be subject to certain symmetries, as, e.g., paritytime symmetry. The study of symmetries may be useful for the basic understanding of light propagation as well as for the specific design of a component or system. Symmetries have thus been investigated in various regards, e.g., from a geometrical-optics point of view ${ }^{2}$, for describing spatial-temporal behavior of a multimode optical oscillator ${ }^{3}$ and for introducing the concept of supersymmetry into the design of optical structures ${ }^{4}$.

A wavefield $u(x, y, z)$ propagating in $z$-direction may be described by its angular spectrum $\tilde{u}\left(k_{x}, k_{y} ; z\right)$ by

$$
u(x, y, z)=e^{i k_{z} z} \iint \tilde{u}\left(k_{x}, k_{y} ; z\right) e^{i\left(k_{x} x+k_{y} y\right)} d k_{x} d k_{y}
$$

where $k_{x}$ and $k_{y}$ are the lateral components of the $\mathrm{k}$-vector, the term $e^{i k_{z} z}$ describes propagation in $z$-direction. Symmetries are due to constraints in the propagation, caused by the geometry of an element/system or by specific properties of the propagation medium. The four symmetries named above may be described mathematically by using the $\mathrm{k}$-vector formalism (Table 1). In the following, we consider several examples of symmetries in the propagation of coherent and incoherent wavefields.

\begin{tabular}{|l|l|}
\hline mirror symmetry (longitudinal inversion) & $k_{z} \rightarrow-k_{z}$ \\
\hline lateral inversion & $k_{x} \rightarrow-k_{x}$ \\
\hline translational symmetry (lateral periodicity) & $k_{x}=k_{x}+\Delta k_{x}$ \\
\hline rotational symmetry & $k_{\theta} \rightarrow k_{\theta}+\Delta k_{\theta}$ \\
\hline
\end{tabular}

Table 1. Symmetries affecting wave propagation. Note, that here just one lateral k-component ( $k_{x}$ and $k_{\theta}$, respectively) is considered.

* Email address: juergen.jahns@fernuni-hagen.de

Fourth International Conference on Applications of Optics and Photonics, edited by

Manuel F. M. Costa, Proc. of SPIE Vol. 11207, 1120702 • (c) 2019 SPIE

CCC code: $0277-786 \mathrm{X} / 19 / \$ 21 \cdot$ doi: $10.1117 / 12.2523115$ 


\subsection{Mirror symmetry (longitudinal inversion)}

When a light ray is reflected from a mirror positioned in an $(x, y)$-plane, its $k_{z}$-component gets inverted: $k_{z} \rightarrow-k_{z}$. Obviously, this is the central equation of reflective optics, in general. A Fabry-Pérot resonator represents an important example, where mirror symmetry plays a role ${ }^{5}$. The field emitted from a two-mirror resonator of length $L$ is periodic in $z$ with period $\Delta z=2 L$, which leads to a discretization of the lateral k-vector components, i.e., with $n$ as an integer number $>0$, one gets:

$$
k_{x}^{2}+k_{y}^{2}= \pm 2 \pi \sqrt{\left(\frac{1}{\lambda}\right)^{2}-\left(\frac{n}{\Delta z}\right)^{2}}
$$
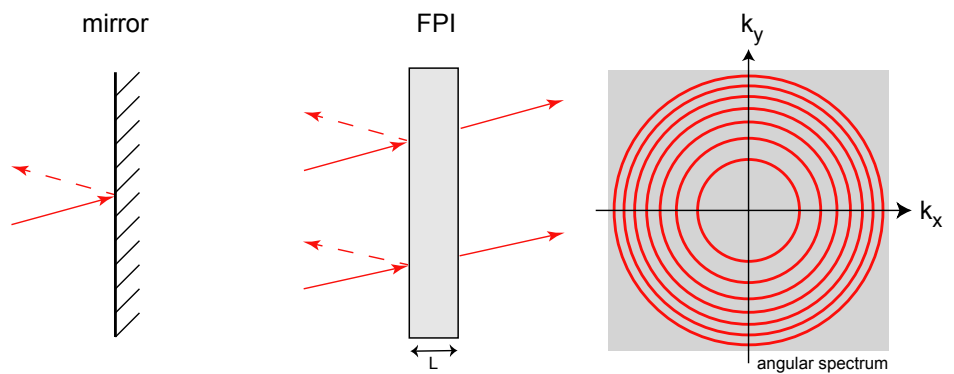

Figure 1. Reflection from a mirror, transmission through a Fabry-Pérot resonator and transmitted angular spectrum.

\subsection{Lateral inversion}

Spatial inversion symmetry is usually considered at the molecular scale, it may have certain consequences for the implementation of nonlinear optical processes ${ }^{6}$. Here, we consider lateral inversion at the systems level which occurs typically in reflective systems, particularly, in systems with tilted optical axis. Examples include the Offner imaging spectrometer 7,8 and microoptical "PIFSO"-systems ${ }^{9}$ (planar integrated free-space optics, see Fig. 2). For the design of a system with a tilted optical axis, lateral inversion (i.e., mirror symmetry with respect to a lateral axis, for example, the $y$ axis) may be used conveniently ${ }^{10}$. If $W_{1}(x)$ (here just $1 \mathrm{D}$ ) represents the phase generated in plane $\mathrm{F}$ by a point source in I, while $W_{2}(x)$ is the phase necessary to focus to diffraction-limited spot in O. Aberrations are given by $W_{a b}(x)=W_{1}(x)-W_{2}(x)$. For $W_{2}(x)=-W_{1}(-x)$, it is $W_{a b}(x)=W_{1}(x)+W_{1}(-x)$ and thus aberrations with antisymmetric terms (i.e., $\left.x, x^{3}, x^{5} \ldots\right)$ cancel out.
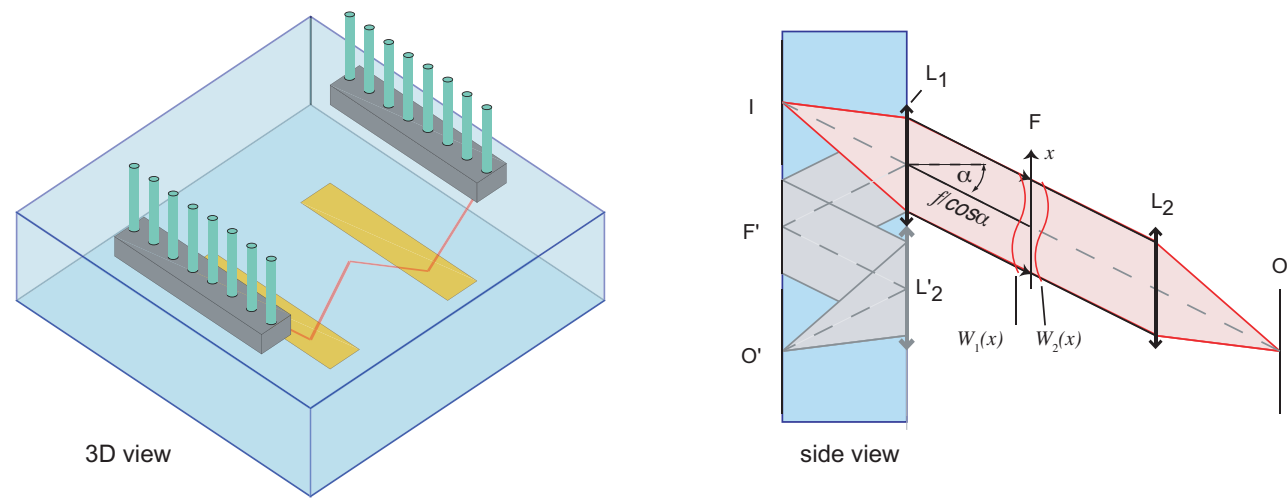

Figure 2. PIFSO system, 3D view and side view. I: input plane, F and F': Fourier planes, $\mathrm{O}$ and O': output planes. The side view shows optical system folded into the substrate and a corresponding (virtual) unfolded $4 \mathrm{f}$-system. 


\subsection{Translational symmetry (lateral periodicity) - coherent light}

Lateral periodicity of a wavefield (described by a discrete angular spectrum) leads to a longitudinal periodicity (at least, for paraxial propagation):

$$
u(x, z=0)=u(x+\Delta x, z=0) \Rightarrow u(x, z)=u(x, z+\Delta z)
$$

A well-known example is the case of Talbot self-imaging 11 in coherent light where a grating is illuminated by a plane wave. "Images" of the grating can be observed at distances which are multiples of the Talbot distance $z_{T}=2 p^{2} / \lambda$ (where $p$ is the lateral period and $\lambda$ is the wavelength (Fig. 3). The self-imaging phenomenon is often referred to for the basic understanding of light propagation and its applications to interferometry.

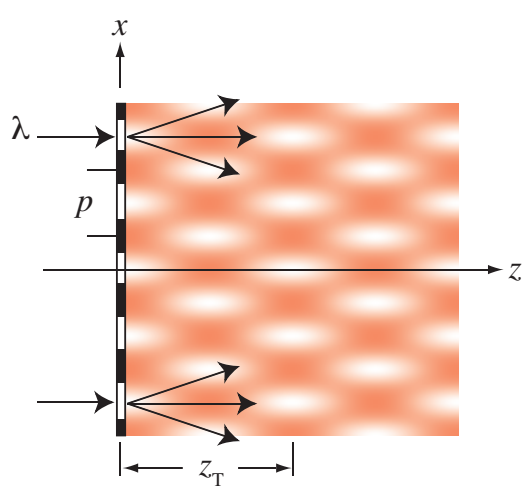

Figure 3. Talbot self-imaging.

A more general case of self-imaging is given by the analysis presented by Montgomery ${ }^{12}$. He asked the question how a wavefield has to be composed in term of its spatial frequencies in order to yield longitudinal periodicity. The answer is that is has to consist of spatial frequencies as given by eq. (2). The conventional Talbot case can be viewed as a special (i.e., the paraxial) case of Montgomery self-imaging.

\subsection{Translational symmetry (lateral periodicity) - partially coherent light}

Coherent self-imaging as described above has a well-known analog for the case of incoherent illumination, known as the Lau effect ${ }^{13,14}$. Here, the setup consists of two gratings, $\mathrm{G}_{1}$ and $\mathrm{G}_{2}$. Upon illumination with monochromatic, yet spatially incoherent light, $\mathrm{G}_{1}$ (an amplitude grating) generates a mutual coherence function in the plane of $\mathrm{G}_{2}$ which is laterally periodic, according to the van Cittert-Zernike theorem ${ }^{15}$. If, under suitable conditions, the period of the mutual intensity function is the same as the period of $\mathrm{G}_{2}$, high-contrast interference fringes are observed at infinity (Fig. 4).

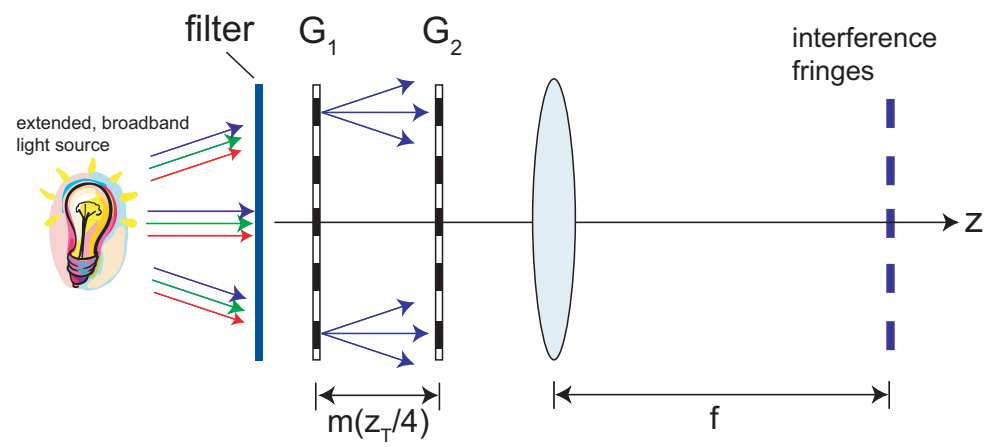

Figure 4. Lau effect, a diffraction experiment with incoherent illumination. 
At this point, it is worthwhile to mention early work on the properties of coherent and partially coherent fields, see, for example ${ }^{16}$. Knowledge of the partially coherent case, i.e. the Lau effect, can be useful for understanding the design of modern lithographic systems. The ever-decreasing size of features in VLSI-chips is partly due to a sophisticated combined optimization of light source and lithographic mask ("mask-source optimization") ${ }^{17}$. Both, light source and mask, exhibit lateral periodicity and may be described mathematically as the 2D case of a Lau interferometer.

\subsection{Rotational symmetry}

Optical elements with rotational symmetry include well-known examples such as lenses and spherical mirrors, described by a quadratic phase function. In recent years, element with linear radial phase, so-called axicons have received much interest ${ }^{18}$. An axicon can be used to generate an elongated focus, a so-called Bessel beam ${ }^{19}$. An axicon with additional azimuthal phase generates a higher-order Bessel beam with orbital angular momentum 20 ("optical vortex"). By additional discretization of the phase function (possible by using a diffractive implementation), additional complexity can be introduced in the wavefield to generate, e.g., multiple vortices ${ }^{21,22}$.
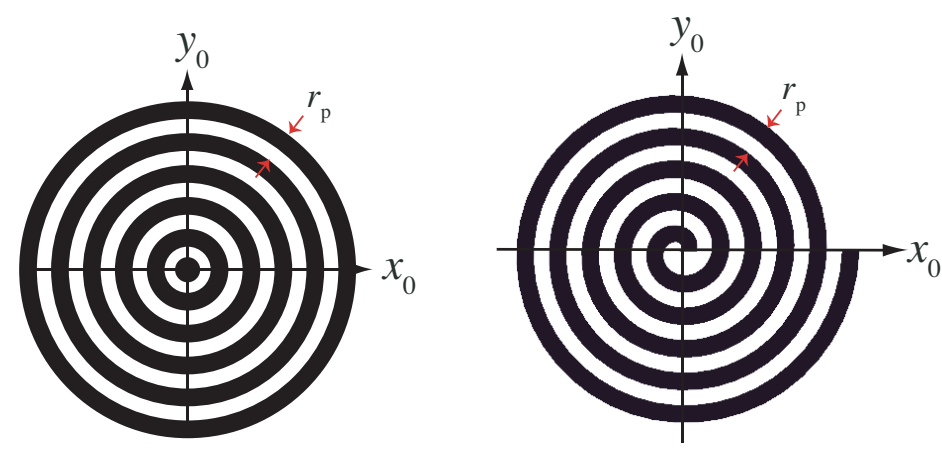

Figure 5. Diffractive axicon and spiral axicon.

\subsection{Combination of translational and rotational symmetry}

Different types of symmetries may be combined to yield wavefields with rather unusual properties. One particular example is shown in Fig. 6, which displays a periodic array of diffractive axicons. In this case of lateral and rotational symmetry combined, Talbot self-imaging is observed, however, the contributions of the vortices in each period add up to yield a spatial variation of the spectral phase 23 .

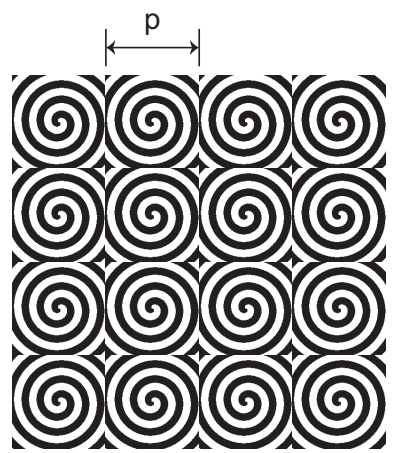

periodicity

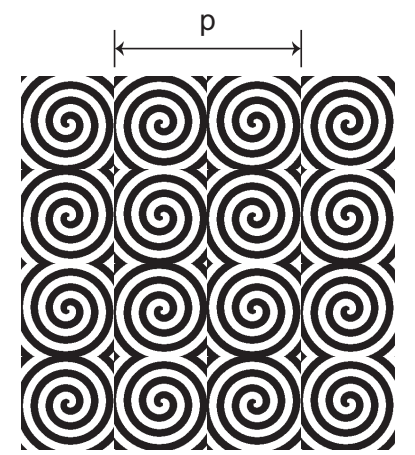

periodicity + mirror symmetry

Figure 6. Periodic arrays of diffractive spiral axicons. Here, $\mathrm{p}$ denotes the lateral period in each of the two patterns. 


\subsection{Combination of lateral and longitudinal periodicity}

The cases of lateral and longitudinal periodicity may be combined to consider the question, how the resulting wavefield looks like. This case is implemented by considering a Fabry-Pérot resonator with two periodically structured mirrors 24 . The analysis shows that stop-bands occur in the spatial frequency spectrum of the transmitted field, similar to the stopbands occuring for photonic crystals, yet here, for structures larger than the wavelength.
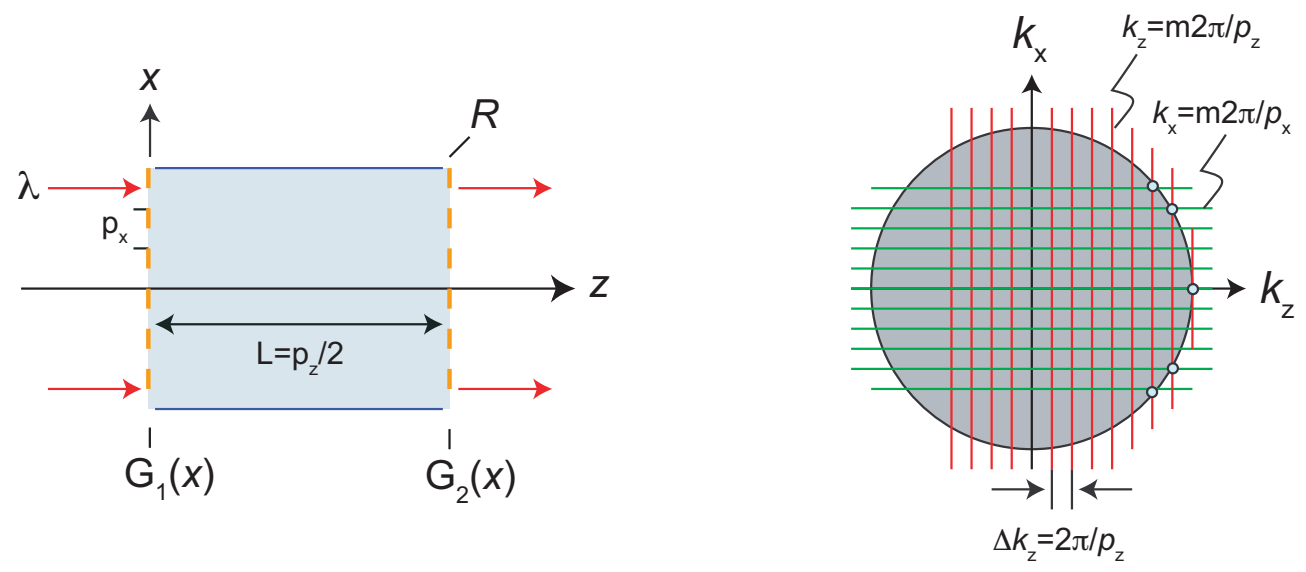

Figure 7. Combination of lateral and longitudinal periodicity: structured resonator and resulting spatial frequencies.

3.

ADDITIONAL CONSIDERATIONS AND CONCLUSION

\subsection{Wigner representation of optical wavefields}

The considerations on optical wavefields and their properties get an additional touch when mapping the signal function into "phase space" by a "Wigner transformation", which combines spatial and spatial frequency coordinates. A comprehensive overview is given in ref. ${ }^{25}$. One particular strength of phase-space optics is that it provides information about the local frequency content of a signal. In connection with symmetry operations as discussed here, the Wigner or phase-space formalism may be helpful to reveal certain properties more easily.

\subsection{Symmetries in data structures}

As mentioned in the introduction, symmetries can be found everywhere in physics, chemistry, biology and mathematics. However, they are of high relevance in computer science, too, and from there, have been of interest for the work on optical computing in the 1980s and 90 s.

A specific example is given by the permutation networks which are underlying the so-called fast algorithms in computing. Fast algorithms like the FFT algorithm by Cooley and Tukey, for example, are based on symmetry properties of the discrete transformation kernel $w_{N}^{n k}=e^{i 2 \pi n k / N}$ using a binary decomposition of the positions $n$ (input) and $k$ (output), e.g., $n=n_{0} 2^{0}+n_{1} 2^{1}+n_{2} 2^{2}$ for $N=8$ and the recursion formula $w_{N}^{2 n k}=w_{N / 2}^{n k}$. This allows one to carry out the calculation of a discrete Fourier transformation recursively using a $\log _{2} N$-architecture 26 .

The computation of the FFT is usually implemented on a so-called Butterfly network (also known as the Banyan network, see Fig. 8a), whose interconnection pattern reflects the symmetry properties just mentioned. The Butterfly network belongs to a wider class of permutation networks which are widely used for computation, sorting and routing applications 27. Butterfly (Banyan), Perfect Shuffle and Crossover network are well-known examples for permutation networks. All of them have been investigated in the context of using optics, in particular, the parallelism of optics in computing and switching ${ }^{28}$. The optical implementation of permutation networks implies certain challenges, mainly because of their space-variant character. Symmetry, however, can be used for an efficient implementation, e.g., by decomposition into "essentially" space-variant sub-operations, as shown, for example for the inverse Perfect Shuffle (Fig. 8b) in ref. ${ }^{29}$. 


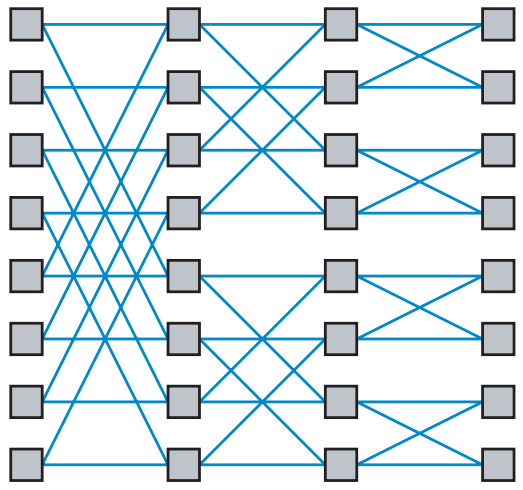

a

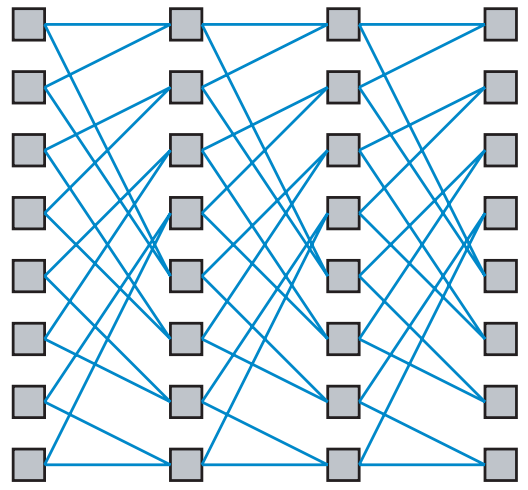

b

Figure 8. a) Butterfly (or Banyan) network. b) Inverse Perfect Shuffle.

\subsection{What symmetry considerations are good for}

The examples presented above indicate the wide variety of situations where symmetries can play a role. From the presentation, it is also obvious, that in some cases, very practical aspects are in the foreground (e.g., with regards to the design of an optical system), while in other cases, more fundamental aspects of light propagation become relevant (e.g., with regards to self-imaging properties). Symmetry considerations may be helpful to predict which effects occur or which cannot occur. They cannot yield definite predictions nor provide a quantitative description.

Symmetries have a high aesthetic value, either for an observer looking at a symmetric pattern or field distribution. Often, the interesting phenomenon occurs for situations with well-defined asymmetries, as it was mentioned already in the introduction. The combination of translational and rotational symmetries described above may serve as an example here. Or, as Pierre Curie, said in 1894: "C'est la dissymétrie qui crée le phénomène" 30,31.

\section{ACKNOWLEDGMENT}

My appreciation goes to members of my group, who participated in various projects as well, as Rüdiger Grunwald (MaxBorn-Institute, Berlin) and his colleagues for a fruitful collaboration. Furthermore, I would like to thank Jorge OjedaCastañeda for his valuable comments. 


\section{REFERENCES}

1. Elliott, J. P. and Dawber, P. G., [Symmetry in Physics], Oxford University Press (1979).

2. Szilagyi, M. and Mui, P. H, "Symmetries in geometrical optics: theory", J. Opt. Soc. Am. A 12, 2753-2759 (1995).

3. Farjas, J., Hennequin, D., Dangoisse, D. and Glorieux, P., "Role of symmetries in the transition to turbulence in optics," Phys. Rev. A 57, 580-584 (1998)

4. Miri, M.-A., Heinrich, M., El-Ganainy, R. and Christodoulides, D. N., "Supersymmetric optical structures," Phys. Rev. Lett. 110, 233902 (2013).

5. Lohmann, A. W., Ojeda-Castañeda, J., and Streibl, N., "Spatial periodicities in coherent and partially coherent fields," Opt. Acta 30, 1259-1266 (1983).

6. Boyd, R. W., [Nonlinear optics, 2nd ed.], Academic Press, Amsterdam (2003).

7. Offner, A., "New concepts in projection mask aligners ," Opt. Eng. 14, 130-132 (1975).

8. Prieto-Blanco, X., Montero-Orille, C., Couce, B. and de la Fuente, R., "Analytical design of an Offner imaging spectrometer," Opt. Express 14, 9156-9168 (2006).

9. Jahns, J. and Huang, A., "Planar integration of free-space optical components," Appl. Opt. 28, 1602-1605 (1989).

10. Jahns, J. and Walker, S. J., "Imaging with planar optical systems," Opt. Comm. 76, 313-317 (1990).

11. Talbot, H. F., "Facts relating to optical science, no. IV," Phil. Mag. 9, 401-407 (1836).

12. Montgomery W. D., "Self-Imaging Objects of Infinite Aperture," J. Opt. Soc. Am. 57, 772-778 (1967).

13. Lau, E., "Beugungserscheinungen an Doppelrastern," Annalen der Physik 6, 417-423 (1948).

14. Jahns, J. and Lohmann, A. W., "The Lau effect (a diffraction experiment with incoherent illumination)," Opt. Comm. 28, 263-267 (1979).

15. Born, M. and Wolf, E., [Principles of Optics, 7th ed.], Cambridge Univ. Press (1999).

16. Lohmann, A. W., Ojeda-Castañeda, J. and Streibl, N., "Symmetries in coherent and partially coherent fields," Opt. Acta 30, 399-402 (1983).

17. Erdmann, A., Fuehner, T., Schnattinger, T., and Tollkuehn, B., "Toward automatic mask and source optimization for optical lithography," Proc. SPIE 5377, (2004).

18. McLeod, J., "The Axicon: A New Type of Optical Element," J. Opt. Soc. Am. 44, $592-597$ (1954).

19. Dyson, J., "Circular and spiral diffraction gratings," Proc. Royal Soc. A, 248, 93-106 (1958).

20. Arlt, J. and Dholakia, K., "Generation of higher-order Bessel beams by use of an axicon," Opt. Comm., 177, 297-301 (2000).

21. Jahns, J., "Continuous and discrete diffractive elements with polar symmetries," Appl. Opt. 56, A1-A7 (2017).

22. Supp, S. and Jahns, J., "Coaxial superposition of Bessel beams by discretized spiral axicons," J. Eur. Opt. Soc. Rapid Publ. 14:18 (2018).

23. Liebmann, M. et al., "Self-imaging of tailored vortex pulse arrays and spectral Gouy rotation echoes," Opt. Lett., 44, 1047-1050 (2019).

24. Jahns, J. and Lohmann, A. W., "Optical wave fields with lateral and longitudinal periodicity," Appl. Opt. 48, 3438-3445 (2009).

25. Testorf, M., Ojeda-Castañeda, J. and Lohmann, A. W., [Selected Papers on Phase-Space Optics], SPIE Milestone Series MS181 (2006).

26. Cooley, J. W. and Tukey, J. W., "An algorithm for the machine calculation of complex Fourier series," 19, 297-301 (1965).

27. Kuck, D. L., [Structure of computers and computations], John Wiley, New York (1978).

28. Smith, D. E., Murdocca, M. J, and Stone, T. W., "Parallel optical interconections," in: J. Jahns, S. H. Lee, eds. [Optical Computing Hardware], Academic Press, Boston (1984).

29. Jahns, J., "Efficient Hadamard transformation of large images," Signal Proc. 5, 75-80 (1983).

30. Curie, P., "Sur la symétrie dans les phénomènes physiques, symétrie d'un champ électrique et d'un champ magnétique", J. Phys. Theor. Appl. 3, 393-415 (1894).

31. Hargittai I., and Hargittai M. [Pierre Curie. In: In Our Own Image], Springer, Boston (2000). 